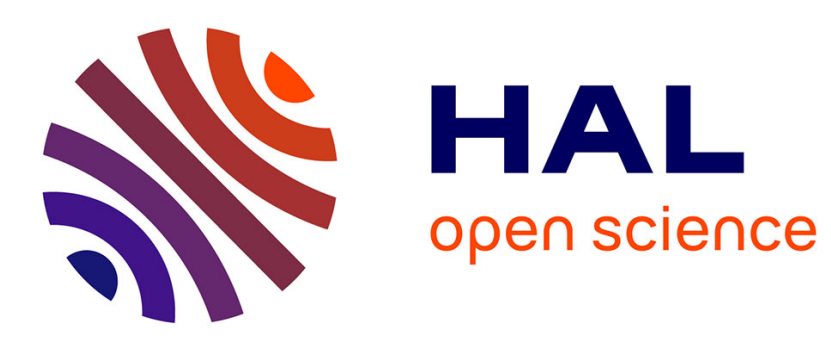

\title{
Note sur une espèce cryptogamique nouvelle pour la France sur différents Pins dont le Pin maritime des Landes
}

Louis Lanier

\section{- To cite this version:}

Louis Lanier. Note sur une espèce cryptogamique nouvelle pour la France sur différents Pins dont le Pin maritime des Landes. Revue forestière française, 1965, 1, pp.34-41. 10.4267/2042/24685 . hal-03389931

\section{HAL Id: hal-03389931 \\ https://hal.science/hal-03389931}

Submitted on 21 Oct 2021

HAL is a multi-disciplinary open access archive for the deposit and dissemination of scientific research documents, whether they are published or not. The documents may come from teaching and research institutions in France or abroad, or from public or private research centers.
L'archive ouverte pluridisciplinaire HAL, est destinée au dépôt et à la diffusion de documents scientifiques de niveau recherche, publiés ou non, émanant des établissements d'enseignement et de recherche français ou étrangers, des laboratoires publics ou privés. 


\title{
NOTE \\ SUR UNE ESPEECE CRYPTOGAMIQUE NOUVELLE POUR LA FRANCE SUR DIFFERENTS PINS DONT LE PIN MARITIME DES LANDES
}

\author{
PAR
}

\section{LANIER}

Ingénieur des Eaux et Forêts

Chargé de Recherches au C.N.R.F.

Dans le cadre d'une étude générale sur un dépérissement inquiétant du Pin maritime qui a été remarqué depuis 1962 dans les Landes, diverses causes ont pu être envisagées.

Les boisements étendus à des zones peu propices de la Grande Lande, l'utilisation massive, après les incendies de 1949, de graines du Portugal, ont pu être invoqués comme causes possibles primaires.

D'autres causes, liées aux précédentes, peuvent également entrer en ligne de compte et parmi celles-ci, certains agents entomologiques ou cryptogamiques.

L'étude proposé a pour but de signaler la présence d'un agent cryptogamique nouvellement répertorié et de faire le point de nos connaissances actuelles sur cet agent.

\section{Introduction - Historique}

Début 1964, nous recevions en provenance de Bourrideys (Gironde) des échantillons de rameaux de Pin maritime landais porteurs de petits chancres sur lesquels se développait un champignon d'apparence extrêmement curieuse (voir photos). 
La détermination effectuée au Muséum National d'Histoire Naturelle nous fournissait Chaenotheca stenocyboides (Nyl.), décrit sur écorce de Pinus pumilio en Bavière (Addenda nova ad lichenographiam european; continuatio nona et tricesima. Exponit W. Nylander. In Flora, 1882, p. 451).

Quelques années plus tôt, PECK (13)* l'avait décrit sur Pinus strobus aux Etats-Unis sous le nom de Caliciopsis pinea et classé parmi les Discomycètes (voir II - Position systématique).

Au cours de l'année 1964, les différents services** alertés sur la présence de ce champignon, dont le classement systématique à proximité des Ascolichens semblait exclure le rôle pathogène, adressaient de nombreux échantillons, toujours liés plus ou moins directement à la présence de petits chancres de rameaux.

Les observations actuelles nous le montrent:

- fréquent sur Pinus pinaster Soland (= Pinus maritima Poir.) à Bourrideys, Cazalis, Saint-Laurent-du-Médoc, Arès, Cestas en Gironde.

- En septembre 1964, nous le relevions à Caudos, Leognan et Ychoux.

- Nous notions à Retjons, toujours dans le massif landais, la présence de chancres sans Caliciopsis.

- Nous le trouvions sur Pinus insignis et Pinus laricio corsicana à l'arboretum des Malgaches.

- Des échantillons reçus à la même époque en provenance du Lubéron (Vaucluse) révélaient également la présence de cet agent sur Pinus halepensis.

Parallèlement à cette étude géographique, l'étude bibliographique permettait de faire le point des connaissances actuelles sur ce sujet. Chronologiquement, les premiers travaux importants qui lui ont été directement consacrés sont dus à FitzPatrick, 1920 (6) qui en a donné une description dans son étude sur les Coryneliacées.

En 1930, Arnaud (1) dans son étude sur les Astérinées, a étudié un échantillon de l'herbier S.P.V.P. de Farlow sur rameaux de Pinus strobus aux Etats-Unis.

La même année, Overholts (11) le signale sur Tsuga canadensis. Les «Genera of Fungi 》 de Clements et Shear (5) de 1931 le prennent comme type du genre Caliciopsis. En 1936, une étude morphologique de son développement est effectuée par Mc CoRMACK (10) et des études expérimentales sur sa culture et sa pathogénicité sont entreprises et relatées par RAY (14).

Les ouvrages principaux de Pathologie Forestière en langue anglaise, celui de Boyce (3) et le tout récent Peace (12) le citent parmi les agents susceptibles de causer sur Pinus strobus des chancres limités alors qu'il est à pev près inoffensif sur les autres espèces.

Le travail de Fitzpatrick, en 1942 (7) décrit l'agent avec plus de détails mais il faut attendre 1963 et Funk (8) pour disposer d'une étude biologique

* Les numéros entre parenthèses renvoient à la bibliographie.

** Pour la région landaise, nous tenons à remercier ici tout spécialement pour son travail de prospection, M. Boulbria de la Station de Recherçes Forestières de Bordeaux. 


\section{CALICIOPSIS PINEA Peck}

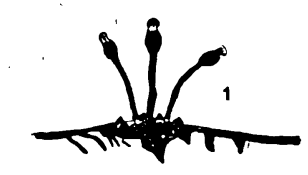

A 1. - Ascocarpes mûrs in situ

2 - Groupe de pycnides à la base d'un jeune ascocarpe
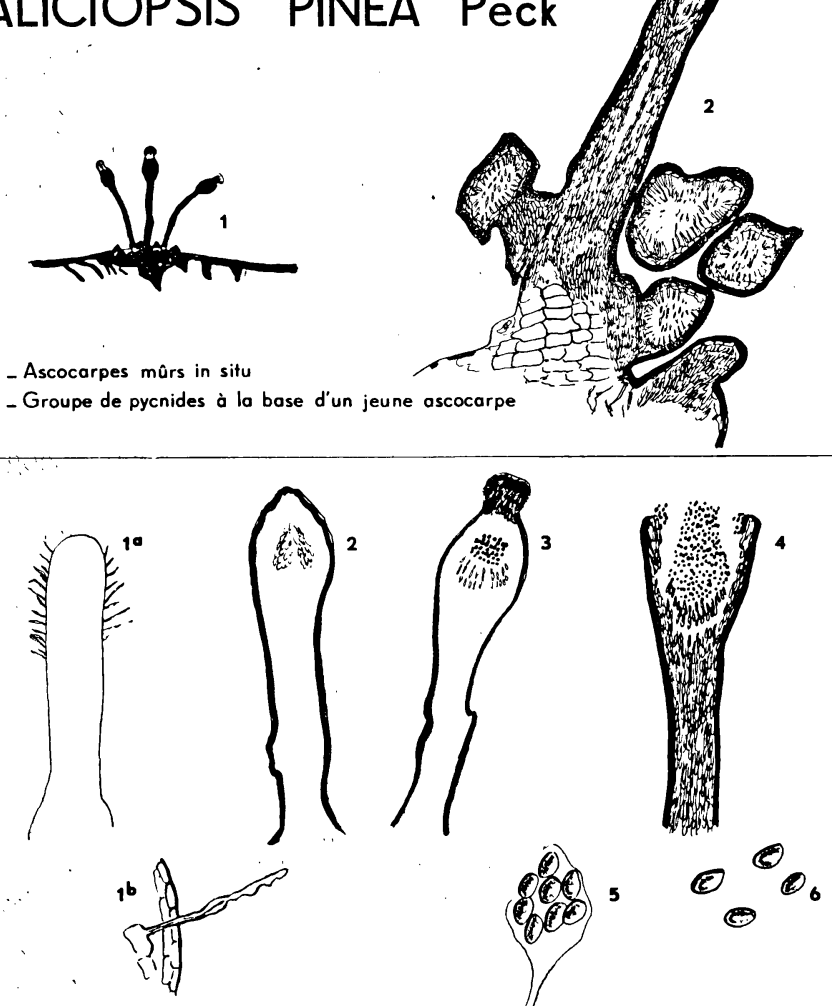

B 1a-Ascocorpe jeune entouré d'hyphes réceptrices 2 - Stade de formation des ascogones

1b_- Hyphes réceptrices (détail)

4 - Libération des ascospores

3 - Formation des ascospores

6 - Ascospores isolées

5 - Asque ò 8 ascospores 
très complète sur le genre Caliciopsis et principalement sur les espèces vivant sur Pinus strobus et Pseudotsuga menziesii.

Les dernières publications de FUNK (9) permettent de préciser quelle est en Amérique du Nord la pathogénicité réelle de Caliciopsis pinea.

\section{I. - Description du champignon}

Le mycélium présent dans le tissu cortical est constitué de cellules cylindriques, allongées, plus ou moins étroites. A la surface de l'hôte se constitue un stroma brunâtre formé par la coalescence des filaments mycéliens. Le stroma prend une forme plus ou moins lobée et se différencie en pycnides et en ascocarpes.

Les pycnides sont petites noires, arrondies à piriformes. A maturité, elles mesurent 100 à $200 \mu$ et renferment à leur intérieur des pycniospores monocellulaires, hyalines à jaunâtres, de 2 à $3 \mu$ de long. On note la présence d'un ostiole, par lequel sont évacuées à maturité les pycniospores réunies en un cordonnet muqueux.

L'ascocarpe mûr, caractéristique, apparaît à l'œil nu comme une sorte d'épine noire constituée par une colonne stromatique, muni à maturité d'un élargissement apical ou sub-apical contenant un simple locule.

Les pycnides sont très souvent groupées à la base de l'ascocarpe (cf. photos). Ce dernier est coriace, brun à noir devenant plus ou moins gélatineux par l'humide. Sa taille peut atteindre 2 à $3 \mathrm{~mm}$ et il est de ce fait parfaitement visible à l'œil nu.

A l'intérieur du locule, les asques peu visibles sont ovales, portés par un long pédicelle et contiennent 8 ascospores brun doré, ellipsoïdes de 5-6 6 3-3,5 $\mu$. Les spores sont libérées par déliquescence de l'extrémité de l'ascocarpe et apparaissent en une protubérance pulvérulente comme l'extrémité d'un petit pinceau. Leur masse brun plus clair à brun rouge est également parfaitement visible à l'œil nu.

Des observations ont suivi le développement de l'ascocarpe qui commence par être une épine pointue entourée d'hyphes réceptrices puis se différencie un locule à l'intérieur duquel se développent les asques. Nos propres observations (voir photos) montrent un développement analogue.

\section{II. - Position systématique}

Les divergences des auteurs quant à la position systématique du genre Caliciopsis proviennent de certains caractères aberrants que présentent les Caliciens (Chadefaud, 1960) (4).

Leurs apothécies restent longtemps closes et figurent des périthèces. Leur premier descripteur, PECK, les considéra comme des Discomycètes proches des Calicieae de FrIes, famille de lichens morphologiquement très voisins. Il fut suivi par Saccardo (16), Rehm (15), Arnaud (1).

De son côté, Fitzpatrick (6) plaça les Caliciopsis parmi les Coryneliaceae c'est-à-dire dans l'ordre des Périsporiales en considérant qu'ils possédaient un 
vrai périthèce, suivi en cela plus tard par Clements et Shear (5). Le même FitzPATRICK (7) les rapprocha des Dothidéales malgré le caractère unituniqué de l'asque. Il semble aujourd'hui que l'on puisse avec Chadefaud placer le genre Caliciopsis dans les Stipités-cupulés rappelant les deux caractères fondamentaux du long pied portant l'apothécie et du locule simulant le périthèce.

Il s'agirait donc en dernière analyse d'un groupe original, très intéressant phylétiquement, intermédiaire entre les Pyrenomycètes et les Discomycètes et présentant des analogies avec certains groupes de lichens ancestraux.

\section{III. - Rôle pathogène}

Quel que soit l'intérêt scientifique d'une classification, le caractère essentiel pour le praticien concerne l'importance du rôle joué par ce champignon. Deux voies peuvent seules permettre de se prononcer avec certitude sur le rôle pathogène d'un tel agent:

$1^{\circ}$ L'observation détaillée des zones d'extension mycélienne est reconnue par des coupes microscopiques effectuées en grand nombre. De cette manière, on peut se rendre compte de la profondeur atteinte et dans une certaine mesure de l'effet provoqué par le mycélium dans les zones corticales des rameaux.

Des centaines de coupes effectuées à travers les écorces à la base des zones occupées par les fructifications de Caliciopsis ont montré que les stromas se forment dans les zones les plus externes de l'écorce. Les filaments isolés pénètrent dans les zones demi-profondes. Les cellules manifestent une certaine hypertrophie, mais sans hyperplasie* apparente.

$2^{\circ}$ En cléfinitive, la voie la plus sûre est l'inoculation suivie de réisolements. Les travaux de RAY (14) et plus récemment, les expérimentations de $\operatorname{FunK}(8,9)$ ont montré le rôle pathogène de Caliciopsis pinea sur Pinus strobus. Le procédé d'inoculation consiste à insérer un petit fragment de gélose contenant le mycélium provenant de cultures pures dans l'écorce de pins sains ou à contaminer par une suspension de spores toujours insérées dans de petites blessures. L'ensemble est recouvert de coton humide. Ces inoculations faites au printemps aboutissent à l'automne de la même année à la formation de petits chancres en zones déprimées identiques à l'aspect observé dans la nature. Au printemps de l'année suivante, il y a apparition de stromas caractéristiques puis de pycnides.

Des expériences comparables effectuées sur d'autres conifères n'ont pas donné de résultats aussi nets.

Les travaux de Funk portant sur l'inoculation de Douglas (Pseudotsuga menziesii) ont montré qu'il n’y avait pas de nécrose à la

* L'hyperplasie désigne l'augmentation, souvent d'origine parasitaire, du nombre des cellules d'un tissu par rapport à la normale. 

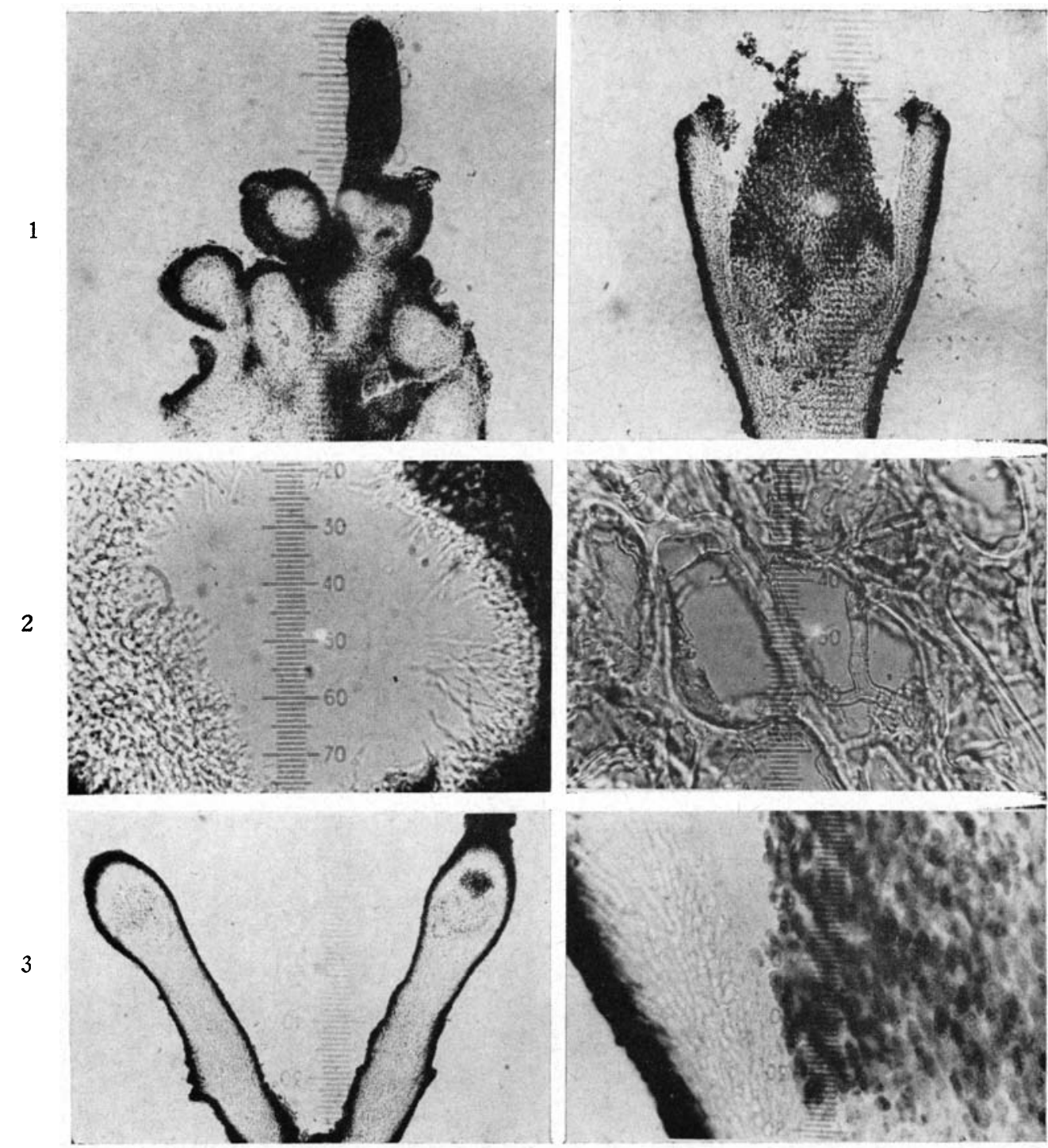

Fig. 1. - Groupe de pycnides à la base d'un jeune ascocarpe (cf. schéma A2).

Fig. 2. - Détail d'une cavité de pycnide.

Fig. 3. - Ascocarpes en cours de maturation.

Fig. 4. - Déliquescence du sommet de l'ascocarpe et libération des ascospores.

Fig. 5. - Mycélium interne - Détail. (Un filament est net à droite du nombre 50 de l'échelle).

Fig. 6. - Asques et ascospores. - Détail.

Pour les photos 1,3 et 4 , une division de l'échelle vaut $11 \mu$.

Pour les photos 2,5 et 6 , une division de l'échelle vaut $2,1 \mu$. 
suite d'inoculations par Caliciopsis pinea. Néanmoins, deux ans après les inoculations, le champignon était toujours présent dans les zones inoculées et montrait des pycnides sans nécrose. En revanche, sur Pinus strobus, les inoculations réussissent à 95 ou $100 \%$ expliquant par là le rôle que peut jouer cet agent sur cette essence.

Les cas d'un champignon se développant sur de petites blessures et donnant lieu à des manifestations pathogènes, sont communs. De nombreux Ascomycètes (Cenangium, Dasyscypha) se comportent ainsi.

L'expérimentation projetée comportera donc:

a) Des témoins sans blessure artificielle,

b) Des témoins avec blessures artificielles,

c) Des arbres avec blessures et inoculations sous forme de mycélium et de spores.

d) Des arbres sans blessures avec inoculations dans des zones lisses.

e) Des arbres sans blessures avec inoculations dans des zones rugueuses (aisselles de branches, aisselles de pseudophylles tombés, etc...).

\section{Conclusions}

Toute conclusion basée sur l'observation externe serait prématurée. Pour l'instant, nous sommes en présence d'un agent nouvellement découvert en France, mais qui y existe peut-être depuis fort longtemps puisqu'il a été découvert sur Pinus pumilio et même sur Abies alba en Allemagne dès le siècle dernier. La découverte de cet agent ne veut nullement dire qu'il soit d'introduction récente.

Cet agent vit abondamment sur différents pins à deux feuilles des régions aquitaines et méditerranéennes. Il se développe à proximité ou sur des chancres dont l'origine est actuellement inconnue.

En revanche, il est expérimentalement connu aux Etats-Unis pour pouvoir causer des chancres à partir de blessures sur certaines espèces de pins.

Il y a donc lieu de se préoccuper de cet agent:

a) Dans le cadre des services de terrain, étant donné qu'il èst très facile à observer, il y a lieu de compléter son repérage de façon à pouvoir cartographier sa répartition actuelle.

b) Dans le cadre de la recherche, des inoculations seront effectuées dès le prochain printemps qui permettront de connaitre son éventuel rôle pathogène dans le cas de nos pins. 
Bibliographie concernant Caliciopsis pinea Peck.

- Caliciopsis stenocyboides Nyl.

Cyphelium stenocyboides Arnold.

Caliciopsis stenocyboides Rehm.

Chaenotheca stenocyboides Nyl.

1. Arnaud (G.). - Les Asterinées - V. (Etude sur les champignons parasites. Caliciacées, Hemisphaeriacées...).

Annales des Epiphyties, 1930, 16, 235-302.

2. Arnaud (G.). - Contribution à l'étude des Fumagines. Annales de l'Ecole Nationale Agronomique, NS, 1912, 12, 23.

3. Boyce (J.-S.). - Forest Pathology.

Mc Graw Hill Book Company, 1948.

4. Chadefaud (M.). - Les végétaux non vasculaires. Masson et Cie, Paris, 1960, 553.

5. Clements (E.) et Shear (L.). - The genera of fungi. H.W. Wilson Co, New-York, 1931.

6. FitzPatrick (H.-M:). - Monograph of the Coryneliaceae. Mycologia, 1920, 12, 206-67.

7. FitzPatRick (H.-M.). - Revisionary studies in the Coryneliaceae. Mycologia, 1942, 34, 464-514.

8. Funk (A.). - Studies in the genus Caliciopsis. C.an. J. of Botany, 1963, 41, 503-43.

9. FUNK (A.). - Extensions of the host ranges and distribution of Caliciopsis species on western conifers. Plant. Dis. Reptr., 1964, 48, 9, 677-78.

10. McCormack (H.-W.). - The morphology and development of Caliciopsis pinea. Mycologia, 1936, 28, 188-196.

11. Overholts (L.-O.). - Mycological Notes for 1928-1929. Mycologia, 1930, 22, 232-46.

12. Peace (T.-R.). - Pathology of Trees and Shrubs. Oxford, Clarendon Press., 1962.

13. Peck (C.). - Annual Report, New-York State Museum, 1880, 33, 32.

14. RAY (W.-W.). - Pathogenicity and cultural experiments with Caliciopsis pinea. Mycologia, 1936, 28, 201-207.

15. Rehм (H.). - Rabenhorst Kryptogamen Flora, 1896, 1, 3, 382.

16. Saccardo (P.-A.). - Syll. Fung., 1899, 8, 825.

- Syll. Fung., 1891, 9, 1073. 